\title{
COLLABORATIVE LEARNING: COLLEGIATE PEDAGOGY UTILIZING WEB CONFERENCING
}

\author{
Joan Ann Swanson ${ }^{1}$, Susan L. Renes ${ }^{2}$ and Anthony T. Strange ${ }^{3}$ \\ ${ }^{I}$ Skidmore College, Saratoga Springs, NY, USA \\ ${ }^{2}$ University of Alaska Fairbanks, Fairbanks, Alaska, USA \\ ${ }^{3}$ Wayland Baptist University, Plainview, Texas, USA
}

\begin{abstract}
This study was completed in two phases, the first of which employed systematic literature analysis of collegiate instructional use of web conferencing followed by a case study of a multi-campus collaborative course utilizing web conferencing. Results provide implications for collegiate educators seeking to employ web conferencing for synchronous instruction and collaborative learning experiences. Additionally, while the study revealed a foundational base for empirical studies related to collegiate pedagogical use of web conferencing, this study also served to expose the need for more sophisticated, generalizable studies regarding web conferencing.
\end{abstract}

\section{KEYWORDS}

Collegiate Instruction, Web Conferencing, Collaboration, Pedagogy

\section{INTRODUCTION}

We exist in places. Places are where we live, work, learn, create, travel to and imagine. While we often think of place in terms of a physical context, Hutchison (2004) notes that places can be a socially constructed reality. He remarks that the "significance of place is often enhanced by the personalities and idiosyncrasies of the individuals who populate a place" (p.11). The place in which learning occurs for today's college students is evolving in large part due to technological advances. The traditional classroom has morphed into any setting in which a screen can capture synchronous or asynchronous instructional activity. This online place is both a locality and special representation of community building and learning. Bringing together individuals in an online setting allows for a reimagining of the learning place. Technology such as web conferencing allows for a mode of social construction which has the ability to move far beyond the constructs of one geographic setting. Advances in modern technology now enable synchronous e-learning through online conferencing, however, empirical studies involving web conferencing for collegiate instruction are still limited (Kang \& Shin, 2015). This study seeks to provide an analysis of how the movement toward web conferencing for educational purposes has impacted the place of collegiate academia.

\section{WEB CONFERENCING IN THE COLLEGIATE SETTING}

Educators are having increased opportunities for synchronous, multimodal communication within academic settings as technological advancements provide web-based communication learning tools. In the 1990s new technologies surfaced on college campuses which allowed for a limited number of users to have real-time communication and collaboration via the internet (Business Matters, 2015). With the introduction of internet services like Skype and iChat, telecommunications became available for free in the 2000s, and by 2010 video conferencing was put to the Cloud and freely available via mobile devices (ezTalks, 2017). The use of video and web conferencing has gained in popularity in both the traditional and online classrooms as an e-learning tool. Web conferencing involves the use of real-time video conferencing software that enables individuals to interact virtually and can be accomplished using any technological device that provides a screen enabling the 
sight of others and sound to hear them. The scope of web conferencing ranges from being the vehicle for a guest speaker, a virtual field trip, group collaboration space, online synchronous instruction time or even virtual office hours. Video and web conferencing can be supplemental for face-to-face courses, or the vehicle to deliver a blended portion of a course or an entire online course.

\subsection{Video Conferencing versus Web Conferencing}

Confusing video and web conferencing is understandable as they deliver similar opportunities. While both involve real-time communication between two or more parties from laptops, desktops or mobile devices, there are subtle differences (See Table 1). Video conferencing has traditionally required special equipment for two-way, high quality video and audio sharing with limited file sharing for specific, controlled audiences. Web conferencing allows for live feed through a web browser that is not limited to a geographical location or number of participants. Web conferencing tends to be less expensive and offers collaborative communication, polls, surveys, whiteboard features and media streaming (Eli, 2017; Erwin, 2019). The course needs will dictate the type of conferencing chosen. For the purposes of this study, web conferencing will be the focus as it has the advantages of being used globally, is less expensive, and offers more academic pedagogical tools.

Table 1. Web and Video Conferencing Contrast

\begin{tabular}{|c|c|c|}
\hline & Web Conferencing & Video Conferencing \\
\hline Purpose & $\begin{array}{l}\text { Content sharing; ideal for face-to-face, impromptu } \\
\text { meetings and delivering large amounts of } \\
\text { information }\end{array}$ & $\begin{array}{l}\text { Communication; ideal at face-to-face } \\
\text { communication for groups and collaboration, } \\
\text { provides real-time }\end{array}$ \\
\hline $\begin{array}{l}\text { Technical } \\
\text { Quality }\end{array}$ & $\begin{array}{l}\text { Internet connection is needed and works across } \\
\text { many geographic locations, sometimes has frozen } \\
\text { images and pixilation due to low bandwidth hence } \\
\text { limiting interaction }\end{array}$ & $\begin{array}{l}\text { High quality images and sound, most similar } \\
\text { to face-to-face, broadcast from one stationary } \\
\text { location on a room-based system, specialized } \\
\text { equipment needed }\end{array}$ \\
\hline Ease of Use & $\begin{array}{l}\text { Authorization not required, only a browser needed, } \\
\text { cheaper than video conferencing, webcast (one-way, } \\
\text { non-interactive) or webinar conference, allows } \\
\text { collaborative communication, polls, surveys and } \\
\text { whiteboard features }\end{array}$ & $\begin{array}{l}\text { Created by an administrator who creates user } \\
\text { accounts for every user and issues personal } \\
\text { credentials for all users }\end{array}$ \\
\hline Tools Needed & Any device connecting to the web & Requires a PC, camera and a microphone \\
\hline Users & Unlimited users & $\begin{array}{l}\text { Limited number of viewers by conferencing } \\
\text { server's capacity }\end{array}$ \\
\hline Application & Presentations, online trainings, distance learning & Regular meetings, discussions \\
\hline
\end{tabular}

(Ch, 2019; Eli, 2017; Erwin, 2019; ezTalks, 2017)

\subsection{Web-based Pedagogy}

Web-based pedagogy involves extending and enriching learning communities beyond the traditional setting. Stevenson and Hedberg (2011) acknowledge tremendous growth in internet-informed pedagogies due to the expansion of web tool availability and cloud computing. Wang and colleagues (2013) suggest that synchronous interaction in the collegiate academic arena aids in bridging both geographic and cultural gaps. Similarly, Muepi (2014) contends web-based technologies are now allowing for expanded world-wide multicultural exchanges and education.

\section{STATEMENT OF THE PROBLEM - CHALLENGES IN HIGHER EDUCATION}

In an era of globalization, higher education is facing many challenges to stay current technologically and keep students engaged and motivated. "Bridging the geographical divide between on campus, off campus, rural, and remote learners has been an ongoing challenge for many universities often resulting in a different learning 
experience based on the mode of study" (Martin \& Broadley2018, p. 55). Knight, et al. (2004) caution the application of banking-model pedagogy and literacy in the online setting could be limiting and detrimental to culturally diverse groups. Martin and Broadley (2018), however propose that the use of distributed learning supported through video and web conferencing will assist in "real time student-centered learning experiences with diverse student perspectives" (p. 55). Additionally, Stevenson and Hedberg (2011) remark that "despite the inherent challenges for adoption within the institution, Web 2.0 technologies do more to unify people across divides - generational, economic, geopolitical and digital - than they do to separate them" (p. 324).

While web conferencing has so much potential, another challenge within higher education is to assist instructors and students to develop the competencies needed to successfully engage in such synchronous activities. Bower, (2011) notes synchronous collaboration competencies need to be developed for multimodal education which includes operational, interactional, managerial, and design abilities. Effectively managing groups and interactions as well as the technology necessitates some level of skill development by all parties. The instructor must then design the learning environment and activities which will support optimal learning. Bower (2011) illustrates that teaching effectively in web conferencing environments does have challenges that can potentially lead to misunderstandings or misuses if institutions do not prepare faculty and students in developing necessary competencies.

The purpose of this study is to understand more fully the place web conferencing currently has pedagogically in the collegiate setting through systematic literature review and a case study utilizing web conferencing. The study was guided by the following research questions:

1. What is the scope of empirical studies about web conferencing in the collegiate instructional setting between 2000 and May 2019?

2. What lessons can be gleaned from a collaborative class case study experience in which web conferencing was utilized?

\section{METHODS}

This study sought to investigate the use of web conferencing for collegiate instruction. The research was conducted into phases using two methodologies: systematic literature review and a collegiate case study. The systematic literature review approach was useful in identifying, selecting and critically analyzing previous empirical studies concerned with the use of web conferencing for instructional purposes (Grant \& Booth, 2009). The second methodology utilized a study approach (Creswell, 2007) and included data collection using course documentation, archival records, interviews, direct observation, participant observation and physical artifacts (Stake, 1995).

\subsection{Phase 1 - Systematic Literature Review}

Data related to web conferencing for collegiate instruction was collected using a contemporary systematic literature review ranging from the year 2000, when video and web conferencing began to be utilized in higher education through the present, 2019. The Ebsco database was used and specific searches were completed using Academic Search Complete, Education Search Complete, ERIC, and PsycINFO. The selection process was guided with the following criteria: papers published between January 2000 and May 2019, papers published in peer-reviewed journals, papers published in English; and papers including the key words web conferencing and college. Relevant, empirical publications were chosen resulting for analysis $(\mathrm{N}=76)$.

\subsection{Phase 2 - Case Study}

Three collegiate instructors, representing three higher education institutions collaborated for a cross-institution experience in an effort to establish a learning community place that stretched beyond the borders of one institution in one locale, to multiple regions and individual representations. This collegiate learning community adopted the guiding framework provided by West and Williams (2017) which includes access, relationships, visions, and functions. 


\subsubsection{Phase 2 - Case Study Participants and Procedure}

The case study participants included students enrolled in higher education course work at three separate institutions located in Alaska, New York, and South Carolina. The participants were registered for a course at their home institution and then met three times throughout the semester for a combined synchronous web conference course session between the three institutions. Students were assigned an introductory activity, pre-class session readings, and activities including post-class reflections where students were able to respond to each other. Each of the three synchronous sessions was led by one of the lead faculty members from the different institutions and conducted through the Zoom platform. Each participant was enrolled in a private WordPress (WordPress.com) website which hosted all course related materials including required media, assignment descriptions and readings, as well as a depository for assignments.

The web conferencing tool Zoom (https://zoom.us/) was used to facilitate the collaborative classes. Students across all three geographic locations joined online at the appointed times, having completed the pre-class session work which served as a basis for the discussions and activities commenced in the hour-long synchronous time. Ground rules were reviewed for discussion times and for ease of facilitating discussion. When a speaker was vocalizing, the Zoom web conferencing program automatically enlarged their image in the center of the screen and all others were minimized in a frame around the perimeter of the screen. At different points in the course, for the purpose of the discussion, screen sharing allowed for documents and images to be viewed as well. All synchronous web conferenced class sessions were also recorded for students and instructors to review.

\section{RESULTS AND ANALYSIS}

\subsection{Phase 1 - Results}

To explore the ways in which web conferencing was being utilized for collegiate instruction, a systematic literature review of educational databases was conducted searching for empirical studies about collegiate web conferencing, which yielded $(\mathrm{N}=76)$ articles with only four of the articles having lead authors publishing more than one publication. These results first indicated a limited number of research specialists focusing in on the use of web conferencing. The methodology utilized was mainly qualitative $(55 \%)$, followed by mixed methods (24\%) and quantitative (21\%). While some studies were mixed methods, using more than one type of method to collect data, the largest amount of studies involved questionnaires or surveys (36\%), followed by case studies $(22 \%)$ and interviews $(12 \%)$. Additionally, other methods noted included observations $(10 \%)$, correlational studies $(9 \%)$, experimental studies $(5 \%)$ and narrative or systematic literature reviews $(6 \%)$. The most frequently used methodology was qualitative, these studies reflected largely on detailed observations and descriptions of data relating to web conferencing in collegiate settings. The data complexities analyzed within the represented systematic literature review were found to be especially useful to describe the human experience in these online environments.

The content area in which the reported studies were associated varied a great deal. While most studies focused upon one course, there were $18 \%$ that were multi-discipline focused, looking globally across a campus. The content areas most represented in web conferencing studies over the past 19 years were Education $(16 \%)$, Medical/Health (12\%), Business (8\%) and Library related (6\%). Other content areas in which web conferencing was studied included: counseling, engineering, geography, history, chemistry, club events, communication, computer programming, criminology, environmental science, math, music, psychology, social work, tutoring, study abroad, and writing.

The most common web conferencing platform or tool reported was Adobe Connect $(33 \%)$, followed by Blackboard Collaborative (16\%), Skype (7\%), and Eluminate Live! (5\%). A total of 17 other tools were also reported however each was only used in one study. The variety of tools utilized indicates there is no singular consensus on the best platform for web conferencing. This is reflective of the proprietary nature of web conferencing tools and that only two seem to have broken into the collegiate technology market in a stronger manner. Since most educational institutions have an established Learning Management System, it would behoove them to further enrich their products with web conferencing tools, similar to the Blackboard Collaborate extension. 
Many of the studies described within this literature review did not report a subject size (50\% of studies reported an $\mathrm{N}=\mathrm{x}$ ), however, a majority of those who did report the number of participants dealt with smaller subject sizes, which is common among case studies, also strongly represented in this review. A total of $62 \%$ of studies reported under 100 subjects, and $44 \%$ had 36 and under for the number of subjects. Considering most college courses contain 40 students or less, the smaller number of subjects in studies involving college courses is commensurate with the typical population.

\subsection{Phase 2 - Results}

Data analysis in phase two involved the researchers reviewing the recordings of the collaborative classes and reviewing the written submissions of students and faculty. Additionally, analysis was conducted within the theoretical frameworks of access, relationships, vision, and function (West and Williams 2017).

The collaborative class was set up to deliberately concentrate on topics which are difficult to discuss. The first class theme focused upon the awareness of our own personal perspectives. Often we hear or believe only a single story about others, when in fact lives are composed of many overlapping stories. The second class theme was about resolving conflict. The last class theme was cultural sensitivity. Students were assigned pre-class work involving watching a media presentation, readings, and completing experiential activities related to the topics. Students were also to come to class having prepared responses to discussion questions, and following the class were required to post reflection pieces. These reflections were then available to be commented upon by other members of class, thus extending interactions. Examples of post-class reflections included the following comments which revealed both vulnerability and growth (See Table 2).

Table 2. Student Reflection Samples

Student 1: I don't think differences should separate and divide people. I think acceptance is a large trend in the world right now and there are a lot of movements trying to increase cohesion between different groups of people. I believe a lot of great opportunities are lost when people decide to divide themselves based on ethnic, cultural, socioeconomic, religious, mental or physical differences.

Student 2: I think that this impacts my work as a teacher because I cannot simply ignore my students' identities. I should not lump all Asian students under one moniker just like I should not lump all people who identify as queer under the idea of gay or being gay. The population of non-cis-straight people is much larger than gay and lesbian, similar to how other populations have branches and subcultures that should be respected. I recognize that I cannot know the entire history of every individual culture that my students may have, but I can put the effort into discussing their cultures and identities with them and researching a little about each of their cultures in order to make sure I am not just assuming they experience life in a certain way or that they identify in a certain way.

Student 3: This experience, the combination of the classroom experiment and my thought processing exercise, evoked feelings of helplessness, a sense of frustration, and feelings of being "outside" the group, or set apart by a difference. It also evoked feelings of empathy as I considered how being blind might impact my own daily life and change the way I live. This experience reminded me of the importance of mindfulness in listening to clients, considering the daily obstacles and roadblocks they may be facing that might be overlooked or missed by anyone not having experience with a disability. As a society, we tend to "other" anyone with a disability. The label of disabled has been used to justify exclusion and derision, in the same way that race, and gender were used previously. ... We view this other able-ness as negative, a weakness, instead of recognizing there is more than one way to navigate the world and experience life that is neither wrong nor less valuable.

Student 4: Like many of the people Chimamanda Ngozi Adichie has encountered in her lifetime, I too was told a single story about other places, whether they were about different continents or different states. I grew up in New York City and as a child I identified anywhere outside of the city as the "countryside." Even suburban areas I dismissed as rural. The term "redneck" was often used in my environment to describe and stereotype people who lived in the southern states. Many people in the city can be very elitist, and I was definitely guilty of this while I was living there. But after high school I was able to travel and go outside of my little city bubble and humanize the places I use to dismiss or overlook. I finally saw that people are more similar than different, but for some reason we like to focus on the differences, maybe to feel better about ourselves and to put ourselves on a pedestal. 


\subsubsection{Access}

On the outset of the course, each member of the community was required to present an "Introduction Selfie" on the course website. This could include their image or anything they felt was representative of them. Additionally, they were to note their home institution, major and why they chose that particular image. This served to humanize and acquaint all course members. Each member of the community had access to each other via course communications. There was also a common meeting place for collaborative sessions, discussions, and access to materials. Regardless of the location of the student, access and opportunity for participation was equal.

The course served to break barriers through web conferencing. For example, in Alaska and other rural areas, web conferencing increases access to higher education. In Alaska, where the communities and the demographics and culture of those communities are so varied, classes such as this increase a student's ability to work following graduation, as they have a better idea of other situations.

\subsubsection{Relationships}

Each person regardless of their locale was an accepted member of the course community and had opportunity to share knowledge, dialogue, and learning experiences. It was established that this was a "safe" community in which members were respectful of each other's differences - especially as topics were broached which challenged personal perspectives and experiences.

\subsubsection{Vision}

The vision for the collaborative learning community was to learn and grow both individually and collectively while examining many topics from multiple understandings, perspectives, and backgrounds. These were hard topics to discuss but enriching to watch the engagement of students across a wide geographic and cultural span.

\subsubsection{Function}

The function of the collaborative class was to socially and digitally create a space in which students could share projects and assignments while also breaching distance and cultural barriers. A Faculty Expectations handout was distributed at the individual institutions that stated the instructor's expectations regarding the class in general, and the interactions between students in the room, as well as distance students. The following ground rules were set up for discussion times: listen with focus and attention; speak without interruption; refrain from giving unsolicited advice or commentary; use I statements; avoid generalizing about people or groups; assume good intentions; and respect difference.

The instructor had to be vigilant about including all of the students in the conversations and activities. If something happened in the classroom that distance students could not see or experience, the instructor explained what just happened so they were not left to feel outside the classroom. The instructors also allowed the distance students plenty of time to talk about their location and how the various concepts being studied might differ elsewhere.

\section{DISCUSSION}

Digital technologies, especially web conferencing, allow students to participate in classes in a highly interactive manner in real-time, using a variety of mobile devices as well as traditional lap top or personal computers. Incorporating these web-based technologies encourages collaboration and communication not only between students and teachers but student-to-student. Additionally, communication and collaboration as noted in this case study potentially spans across campuses as well. Web conferencing as described in both the systematic literature review and case study serves to increase accessibility and opportunity for diversity in learning experiences.

The web conferencing experience in the collaborative class enriched students' perspectives and exposed students to a diverse range of fellow students and situations. One instructor noted, "I think in Alaska students get a much richer experience than they would otherwise; learning from others who are in remote locations, they come to appreciate the differences in access to health care and limitations in travel, etc. as well as the particular benefits and problems of living remotely in Alaska." Another instructor noted a student from New 
York City commented on how they realized their perspectives of other students prior to the collaborative class discussions were inaccurate.

The nature of a course being hosted through web conferencing, such as the collaborative class case study, ultimately adds pedagogical and methodological variety to instruction. Faculty need to focus more on student-centered and active learning activities in which collaborative learning can occur within groups and across groups locally and remotely. More authentic tasks and problem-solving via web technology connections potentially promotes developing 21 st Century skills for a more global society.

In addition to the benefits of collaboration, the collaborative web conferencing class was financially feasible for each institution with no extra cost for individual students. Since all interactions were web-based, using Zoom and a WordPress website, there were no extra costs and all tuition remained at the home institution for each school.

\subsection{Suggestions for Organizing Web Conferencing and Collaborative Experiences}

The faculty involved in the collaborative course experience which utilized web conferencing were able to tackle this new experience together, strategizing procedures and protocols, sharing plans, concepts, and sharing the instructional presentation load. Recommendations for web conferencing and collaborative experiences should first be clear with their goal or objective - beyond just an opportunity to use new technology. In this case study, there were many logistical hurdles that were conquered, but it took time and organization centered upon the purpose for the experience. Since the technological tools will vary across institutions, the faculty involved in this study recommends that instructors practice prior to the first instructional session. Step-by-step instructions on how to access the tools and materials should be provided. Also, on the outset of the course, instructors should establish protocol for how the class will function, ground rules for interactions, and lastly provide a trouble shooting resource and technical support person for assistance.

\section{CONCLUSION}

The systematic literature review revealed many holes in the knowledge base about web conferencing as a collegiate pedagogical tool. Even though it has been actively used since the year 2000, the documentation about the foundation for pedagogical practices is fragmented. Empirical studies are quite limited over the 19-year span, with very few quantitative studies. Additionally, there has been little analysis of the pedagogical effectiveness of web conferencing in singular disciplines. While case studies and singular uses within disciplines can serve to provide individual in-depth knowledge about experiences using web conferencing, these results are more difficult to generalize across other collegiate situations. This study served to expose the need for more sophisticated, generalizable studies regarding web conferencing.

One limitation of this study could be the exclusion of literature review related specifically to video conferencing. The researchers focused upon web conferencing as a more amenable vehicle for synchronous pedagogy. However, future studies will encompass video conferencing as well.

An instructor, teaching in two formats (face-to-face and simultaneously web conferencing) must constantly being paying attention to the local and remote students. Instructors and higher education institutions have to ask themselves why they are instituting web conferencing. Why are they seeking to offer this option? It's optimal to be able to offer options that allow students to take classes the way they learn the best. Distance or web conferencing pedagogy adds a new dimension to education, one that increases access and therefore opportunities to many students who might not otherwise be able to take advantage of education past the secondary level. Web conferencing and collaborative classes also allow for the opportunity for students across geographic and cultural spans to interact, thus promoting and extending diversity acceptance. The goal of web conferencing and collaborative classes is construct a new place of social reality which increases learning and moves far beyond the physical constructs of one geographic setting and any singular pedagogy. Berens (2012) concludes, "It doesn't matter to me if my classroom is a little rectangle in a building or a little rectangle above my keyboard. Doors are rectangles; rectangles are portals. We walk through." Collegiate educators are challenged to venture into a new place for learning experiences, including web conferencing and collaborative teaching situations. 


\section{REFERENCES}

Berens, K. I., 2012. The new learning is ancient. Retrieved from http://kathiiberens.com/2012/12/03/ancient/

Bower, M. 2011. Synchronous collaboration competencies in web-conferencing environments - their impact on the learning process. Distance Education, Vol.32, No. 1, pp 63-83.

Business Matters, 2015. The history of video conferencing. Retrieved from https://www.bmmagazine.co.uk/tech/historyvideo-conferencing/

Ch, R. 2019. What is the difference between web conferencing and video conferencing? Retrieved from https://www.quora.com/What-is-the-difference-between-web-conferencing-and-video-conferencing

Creswell, J. W. 2007. Qualitative inquiry and research design: Choosing among five approaches. Sage, Thousand Oaks, CA.

Eli, B. 2017. Understanding the difference between web conferencing and video conferencing. Retrieved from https://www.eztalks.com/video-conference/difference-between-web-conferencing-and-video-conferencing.html

Erwin, J. 2019. What is the difference between video and web conferencing? Premiere Global Services, Inc. Retrieved from https:/www.pgi.com/resources/articles/what-is-the-difference-between-video-and-web-conferencing/

ezTalks, 2017. A Brief History of Video Conferencing from 1964 to 2017. Retrieved from https://www.eztalks.com/videoconference/history-of-video-conferencing.html

Grant, M. J. \& Booth, A., 2009. A typology of reviews: An analysis of 14 review types and associated methodologies. Health Information \& Libraries Journal, Vol. 26, No. 2, pp 91-108.

Hutchison, D., 2004. A natural history of place in education. Teachers College Press, New York, NY

Kang, M. \& Shin, W.S. (2015). An empirical investigation of student acceptance of synchronous e-learning in an online university. Journal of Educational computing Research, Vol.52, No.4, pp 475-495.

Knight, M. G., Dixon, I. R., Norton, N. E. L., \& Bentley, C. (2004). Extending learning communities: New technologies, multiple literacies, and cultural blind pedagogies. The Urban Review, Vol. 36, No. 2, pp101-118.

Martin, R., \& Broadley, T. 2018. New generation distributed learning: Models of connecting students across distance and cultural boundaries. Austrailian and International Journal of Rural Educaiton, Vol.28, No. 1, pp 55-72.

Mupepi, M. G. 2014. A schematic description of the nature of video-conferencing and Internet exchange: Enhancing global understanding. International Journal of Web-Based Learning and Teaching Technologies, Vol.9, No.1, pp 33-40.

Stake, R. 1995. The art of case study research. Sage, Thousand Oaks, CA.

Stevenson, M. \& Hedberg, J., 2011. 'Head in the clouds: a review of current and future potential for cloud-enabled pedagogies'. Educational Media International, Vol. 48, No. 4, pp. 321-333

Wang, C. X., Jaeger, D., Liu, J., Guo, X., \& Xie, N. (2013). Using synchronous technology to enrich student learning. TechTrends, Vol.57, No.1, pp 20-25.

West, R. E. \& Williams, G. S. 2017. "I don't think that word means what you think it means": A proposed framework for defining learning communities. Educational Technology Research Development, Vol.7, pp 1-14. https://doi.org/10.1007/s11423-017-9535-0 